\title{
MOLECULAR DYNAMIC SIMULATION ON THE THERMAL CONDUCTIVITY OF NANOFLUIDS IN AGGREGATED AND NON-AGGREGATED STATES
}

\author{
S. L. Lee ${ }^{1}$, R. Saidur ${ }^{2}$, M. F. M. Sabri ${ }^{1}$, and T. K. Min $^{3}$ \\ ${ }^{1}$ Department of Mechanical Engineering, University of Malaya, \\ Kuala Lumpur, Malaysia \\ ${ }^{2}$ Centre of Research Excellence in Renewable Energy (CoRE-RE), \\ King Fahd University of Petroleum and Minerals (KFUPM), Dhahran, \\ Kingdom of Saudi Arabi \\ ${ }^{3}$ School of Physics, Universiti Sains Malaysia, Pulau Penang, Malaysia
}

Nanofluids are engineered by suspending nanoparticles in convectional heat transfer fluids to enhance thermal conductivity. This study is aimed at identifying the role of nanoparticle aggregation in enhancing the thermal conductivity of nanofluids. Molecular dynamic simulation with the Green Kubo method was employed to compute thermal conductivity of nanofluids in aggregated and non-aggregated states. Results show that the thermal conductivity enhancement of nanofluids in an aggregated state is higher than in a non-aggregated state, by up to $35 \%$. The greater enhancement in aggregated nanofuids is attributed to both higher collision among nanoparticles and increases in the potential energy of nanoparticles.

\section{INTRODUCTION}

Nanofluids are engineered by suspending nanoparticles, such as oxides or metals, into conventional heat transfer fluids to enhance thermal transport. Nanofluids with higher thermal conductivity than base fluids have attracted much interest from many researchers for the past few decades. Experimentally, nanofluids show enhanced thermal conductivity [1-5]. However, the key mechanisms governing enhanced thermal conductivity have not been determined to date. Several possible mechanisms are currently being explored by researchers: the Brownian motion of nanoparticles [6-9], molecular-level layering at the solid-liquid interface [6, 10-13], microconvection induced by Brownian motion of nanoparticles [7, 14-16], and the effect of nanoparticle aggregation [6, 17-19]. Keblinski [20] concluded that, based on their research and analysis of experimental data [9, 12, 13, 17, 19], it was clearly demonstrated that nanoparticle aggregation is the only mechanism capable of explaining the thermal conductivity of nanofluids.

Received 13 July 2014; accepted 7 November 2014.

Address correspondence to R. Saidur, Department of Mechanical Engineering, University of Malaya, 50603 Kuala Lumpur, Malaysia. E-mail: saidur@kfupm.edu.sa; saidur912@yahoo.com

Color versions of one or more of the figures in the article can be found online at www.tandfonline. com/unht. 


\begin{tabular}{|llll|}
\hline \multicolumn{3}{|c|}{ NOMENCLATURE } \\
$a$ & acceleration, $\mathrm{m} / \mathrm{s}^{2}$ & $v$ & velocity, $\mathrm{m} / \mathrm{s}$ \\
$E$ & per atom energy for kinetic and & $V$ & volume, $\mathrm{m}^{3}$ \\
& potential, $\mathrm{J}$ & $\Phi$ & Lennard-Jones potential, $\mathrm{J}$ \\
$F$ & force, $\mathrm{N}$ & $\varepsilon$ & interaction strength, $\mathrm{J}$ \\
$h$ & average partial enthalpy, $\mathrm{J}$ & $\sigma$ & interatomic length scale, $\mathrm{m}$ \\
$J$ & heat current, $\mathrm{J} \cdot \mathrm{m} / \mathrm{s}$ & $\sigma$ & nanoparticle volume fraction, $\%$ \\
$k$ & thermal conductivity, $\mathrm{W} / \mathrm{m} \cdot \mathrm{K}$ & $\mathrm{Subscripts}$ & \\
$k_{B}$ & Boltzmann constant, & ar & argon \\
& 1.38 $\times 10^{-23} \mathrm{~J} / \mathrm{K}$ & $\mathrm{cu}$ & copper \\
$k e$ & kinetic energy, $\mathrm{J}$ & $\mathrm{f}$ & base fluid \\
$m$ & mass, $\mathrm{kg}$ & $\mathrm{i}$ & particle $\mathrm{i}$ \\
$N$ & total number of particles & $\mathrm{j}$ & particle $\mathrm{j}$ \\
$\mathrm{pe}$ & potential energy, $\mathrm{J}$ & $\mathrm{nf}$ & nanofluid \\
$r$ & displacement between particles, $\mathrm{m}$ & $\mathrm{p}$ & nanoparticle \\
$T$ & temperature, $\mathrm{K}$ & $\alpha$ & species $\alpha$ \\
\hline
\end{tabular}

Ideally nanofluids have well-dispersed nanoparticles in base fluids, but it is experimentally proven that nanofluids are in an aggregated state [5, 21-24]. This is because nanoparticles suspended in base fluid are under the influence of forces Brownian and the Van der Waals. Thus, nanoparticles may tend to aggregate under these forces. Aggregated nanoparticles form linear chains or percolating networks embedded in large pockets of base fluids, to create an additional conduction path for the thermal conductivity of nanofluids [18, 19, 25]. Furthermore Eapen [24, 26], who conducted a large number of experiments on this subject, demonstrated that thermal conductivity in nanofluids is controlled by the Hashin-Shtirkman (HS) bounds. The lower HS bound corresponds to Maxwell's prediction and refers to well-dispersed nanoparticles, whereas the upper HS bound represents linear chains such as nanoparticles [24]. Enhanced thermal conductivity is strongly dependent on the geometrical configuration of nanoparticles, whether they remain dispersed in the base fluid, form linear chain-like configurations, or are in intermediate configurations. This explains the discrepancies that exist among reported experiments, because the results from several experiments involving well-dispersed nanoparticles agree well with Maxwell's prediction [27-29]. However, some researchers report anomalous thermal conductivity enhancement far beyond Maxwell's prediction [5, 22, 30-32]. One should bear in mind that the clumping of large aggregations of nanoparticles will not provide enhanced thermal conductivity. Eastman et al. [33] commented that such clumping would most likely settle out of the fluid due to gravity, especially at low volume fraction, and create large regions of particle-free base fluid with higher thermal resistance. A similar study was experimentally performed by Philip et al. [34] on nanofluids with $\mathrm{Fe}_{3} \mathrm{O}_{4}$ nanoparticles dispersed in kerosene. The alignment of magnetic nanoparticles was controlled by varying externally applied magnetic strength. Maximum thermal conductivity was observed when nanoparticles aggregated into linear chains and were uniformly dispersed in the base fluid; decrease in thermal conductivity was observed following clumping of nanoparticle chains in the base fluid. This provides strong experimental evidence in support of the aggregation of nanoparticles, with linear chains or percolating structures playing an important role in 
enhancing thermal conductivity in nanofluids. Moreover, Hong and Kim [35] and Shalkevich et al. [36] experimentally demonstrated that gelled nanofluids with aggregated nanoparticles produced markedly greater enhancement of thermal conductivity than fluidic nanofluids, with well-dispersed and freely moving nanoparticles.

Nanoparticle aggregation in most experimental works on nanofluids is always unknown and is difficult to change in a controlled manner. Referring back to previous experimental studies, aggregation of nanoparticles can be controlled in two ways. The first method is to have nanofluids with magnetic nanoparticles and their alignment controlled by an external magnetic field [34]; the second is to control the electrical double layer of nanoparticles by the use of additives to produce aggregation or good dispersal $[35,36]$. However, experimental studies can only provide quantitative but not qualitative results at the microscopic level. Therefore, molecular dynamic (MD) simulation is applied by many researchers to complement experimental results at the atomistic level [37-39], but this can be extremely difficult in practice. Many MD simulations have been successful in predicting the thermal conductivity of nanofluids, but the majority are based only on a single nanoparticle in a simulation box [6, 40-48]. The shortcoming of such models is that nanofluids with a single nanoparticle relate only to well-dispersed nanoparticles, where aggregation and collision among particles is not taken into consideration. Therefore, MD simulation on nanofluids with multinanoparticles is required to include the effect of aggregation and collision, and to create situations that are close to the actual conditions. To the best of the current authors' knowledge, there are a limited number of MD simulation studies on the role of nanoparticle aggregation modeling nanofluids with multinanoparticles. Kang [49] applied MD and successfully demonstrated that nanoparticle aggregation induces higher thermal conductivity in nanofluids and that thermal conductivity enhancement is strongly dependent on the configuration of nanoparticle aggregation. However, no further microscopic details were revealed in their MD simulations. Due to lack of microscopic detail on nanoparticle aggregation from existing experimental works and MD simulations, the authors aimed to employ MD for further investigations. In this paper, equilibrium MD (EMD) using the Green Kubo method was performed to study the thermal conductivity of nanofluids in aggregated and nonaggregated states. A nanofluid system with argon liquid and copper nanoparticles was modeled. Multinanoparticles were placed in the simulation box to simulate aggregated and nonaggregated states. By analyzing the components of heat current by Green Kubo formalism, microscopic details of thermal conductivity enhancement in both the aggregated and nonaggregated state can be described.

\section{METHODOLOGY}

MD describes a set of atom trajectories purely based on Newton's second law, $\mathbf{F}=m \cdot \mathbf{a}$, where $\mathbf{F}$ is the force exerted on the particle, $m$ is its mass, and a is its acceleration. By giving the initial condition of masses and force, the position and velocity of the atoms can be determined. The total force acting on the atoms is derived from the interatomic potential and thermostat. The Green Kubo method [50] is an EMD approach to obtain thermal conductivity from the heat current autocorrelation function (HCACF):

$$
k=\frac{1}{3 V k_{B} T^{2}} \int_{0}^{\infty}\left\langle\mathbf{J}_{i}(t) \mathbf{J}_{i}(0)\right\rangle d t
$$


where $k$ is thermal conductivity, $V$ is the volume of the simulation box, $T$ is the system temperature, $k_{B}$ is the Boltzmann constant, and $\langle\mathbf{J}(t) \mathbf{J}(0)\rangle$ is HCACF. HCACF must decay to zero within the integral time/correlation length $(M)$ to produce convergence of data. Thus, determination of correlation length is important in order to ensure sufficient time for the autocorrelation function to decay to zero. Overlong correlation length may result in a spurious value for transport coefficient calculation, while inadequate correlation length may generate premature data [51]. The heat current is given by

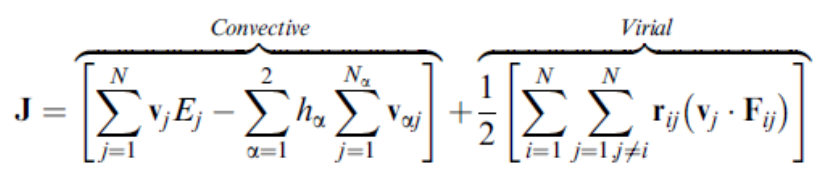

where $\mathbf{v}_{j}$ is the velocity of particle $j ; E_{j}$ is per-atom energy for kinetic and potential; $h_{\alpha}$ is the average partial enthalpy of species $\alpha ; \mathbf{r}_{i j}$ and $\mathbf{F}_{i j}$ are the displacement and interacting forces between particles $i$ and $j$, respectively; and $N$ is the total number of particles. The first term is referred to as the convection of particles, which is internal energy with the summation of kinetic energy and potential energy. The second term is referred to as collision, which is interaction between particles or the work done by the stress tensor. Average partial enthalpy is average kinetic energy, potential energy, and average collision per particle:

$$
\begin{gathered}
h_{\alpha}=\frac{1}{N_{\alpha}} \sum_{j=1}^{N_{\alpha}}\left(E_{j}+\mathbf{r}_{j} \cdot \mathbf{F}_{j}\right) \\
h_{\alpha}=\frac{1}{N_{\alpha}} \sum_{j=1}^{N_{\alpha}}\left[\left(k e_{j}+p e_{j}\right)+\mathbf{r}_{j} \cdot \mathbf{F}_{j}\right]
\end{gathered}
$$

Average partial enthalpy is important to determine the thermal conductivity of multicomponent systems [52]; partial enthalpy for a single-component system is zero. However, partial enthalpy is not zero for multicomponent systems and must be subtracted from the convective term. Therefore, for simulation of thermal conductivity of nanofluids, the proper definition of partial enthalpy is necessary so that it does not lead to an anomalously high thermal conductivity enhancement. Subtraction of partial enthalpy has always been overlooked in a number of previous EMD studies on thermal conductivity of nanofluids, and thus an order of magnitude larger for thermal conductivity was observed.

\section{MODEL}

In this work, a nanofluid system with argon base fluid and suspended copper nanoparticles was developed. Argon liquid was selected as the base fluid due to well-matched interatomic potential with the experimental data and less computation time compared with other more complex base fluids like water. A simulation box, which consisted of argon atoms with face-centered cubic (FCC) lattice constant $5.72 \AA$ and spherical regions, was created by placing copper atoms with FCC lattice 


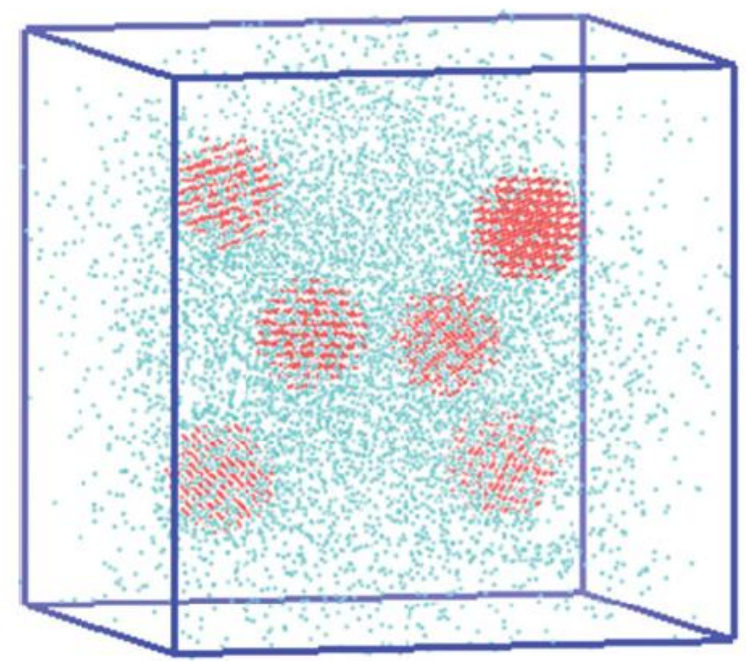

Figure 1. Simulated nanofluid system consisting of argon liquid (blue) and copper nanoparticles (red).

constant $3.62 \AA$. The nanofluid system with multinanoparticles was modeled at a temperature of $86 \mathrm{~K}$. The volume fraction of the nanofluid system was controlled by a varying number of nanoparticles with a fixed diameter of $20 \AA$. The simulation box size was 12 lattices of argon along three axes. Figure 1 shows a simulated nanofluid system with multinanoparticles. According to a finite size study carried out by Sarkar and Selvam [41], the simulation result of pure argon was in good agreement with experimental data when the number of argon atoms was more than 500. Therefore, the number of argon atoms in this study was set at a figure of over 6,380 throughout the simulations.

To study the effect of aggregation on thermal conductivity enhancement, five sets of nanofluid systems with five different volume fractions $(2.59 \%, 3.89 \%$, $5.18 \%, 6.48 \%$, and $7.77 \%$ ) were modeled in both aggregated and nonaggregated and states. Both systems have the same numbers but with different positions of nanoparticles. Table 1 shows the number of atoms of copper and argon in five sets of nanofluids with different nanoparticle volume fractions. One set with pure argon

Table 1. Number of atoms in aggregated and nonaggregated $\mathrm{Cu}-\mathrm{Ar}$ nanofluid system

\begin{tabular}{lccccc}
\hline $\begin{array}{l}\text { Volume } \\
\text { fraction (\%) }\end{array}$ & $\begin{array}{c}\text { Number of } \\
\text { nanoparticles }\end{array}$ & $\begin{array}{c}\text { Number of } \\
\text { argon atoms }\end{array}$ & $\begin{array}{c}\text { Number of } \\
\text { copper atoms }\end{array}$ & $\begin{array}{c}\text { Total } \\
\text { atoms }\end{array}$ & $\begin{array}{c}\text { Size of simulation } \\
\text { box }\left(\AA^{3}\right)\end{array}$ \\
\hline 0.00 & 0 & 6,912 & 0 & 6,912 & 323,393 \\
2.59 & 2 & 6,742 & 702 & 7,444 & 7,706 \\
3.89 & 3 & 6,651 & 1,055 & 7,976 \\
5.18 & 4 & 6,564 & 1,412 & 8,274 \\
6.48 & 5 & 6,467 & 1,807 & 8,556 \\
7.77 & 6 & 6,380 & 2,176 & \\
\hline
\end{tabular}


liquid was simulated for comparison purposes with other nanofluid systems. Table 2 shows the configurations of aggregated and nonaggregated nanoparticles. Argon

Table 2. Configurations of nanofluid systems in nonaggregated and aggregated states

\begin{tabular}{|c|c|c|c|}
\hline Volume fraction $(\%)$ & $\begin{array}{c}\text { Number of } \\
\text { nanoparticles }\end{array}$ & Nonaggregated state & Aggregated state \\
\hline 2.59 & 2 & & \\
\hline 3.89 & 3 & & \\
\hline 5.18 & 4 & & \\
\hline 6.48 & 5 & & \\
\hline 7.77 & 6 & & \\
\hline
\end{tabular}


liquid is excluded in the display of configurations in order to provide a clearer overview of nanoparticle positions. For the nonaggregated state, the nanoparticles were placed so that they had no contact with each other within the time domain. A larger time domain is required to enable aggregation to take place in nanofluid systems, but this is unrealistic for a MD simulation [49]. Thus, for the aggregated state, the nanoparticles were initially placed so as to be in contact with or close to each other to enable aggregation within the time domain.

Lennard-Jones (L-J) potential [53] was employed for interatomic interaction between argon-argon and copper-copper, and is described by

$$
\begin{gathered}
\Phi\left(r_{i j}\right)=4 \varepsilon\left[\left(\frac{\sigma}{r_{i j}}\right)^{12}-\left(\frac{\sigma}{r_{i j}}\right)^{6}\right] \quad\left(r_{i j}<r_{\text {cutoff }}\right) \\
\Phi\left(r_{i j}\right)=0 \quad\left(r_{i j} \geq r_{\text {cutoff }}\right)
\end{gathered}
$$

where $r_{i j}$ is the distance between atoms $i$ and $j, \varepsilon$ represents interaction strength, and $\sigma$ is an interatomic length scale. The cutoff radius of $2.8 \sigma_{\text {ar-ar }}$ was chosen because thermal conductivity is independent at this cutoff point. L $-\mathrm{J}$ potential parameters for argon-argon and copper-copper are shown in Table 3. The Lorentz-Berthelot mixing rule [54] was used to determine the interaction between copper and argon:

$$
\begin{gathered}
\varepsilon_{\mathrm{ar}-\mathrm{cu}}=\sqrt{\varepsilon_{\mathrm{ar}} * \varepsilon_{\mathrm{cu}}} \\
\sigma_{\mathrm{ar}-\mathrm{cu}}=\frac{\sigma_{\mathrm{ar}-\mathrm{ar}}+\sigma_{\mathrm{cu}-\mathrm{cu}}}{2}
\end{gathered}
$$

The time step for the simulations was $4 \mathrm{fs}$, and the nanofluid system was equilibrated for $100 \mathrm{k}$ time steps under canonical ensemble (NVT) ensemble. The equilibration process was performed to insure that the system equilibrated at the desired temperature and the equilibration steps were ignored for thermal conductivity calculation. An additional $1,000 \mathrm{k}$ time steps were run to allow the fluctuation of HCACF under microcanonical ensemble (NVE). HCACF was recorded at a correlation length of $20 \mathrm{ps}(M=5,000)$. The thermal conductivity of each set of nanofluid systems was obtained by averaging five independent simulation runs with different initial velocities; $10 \mathrm{CPU}$ was occupied for each simulation run. All simulations were performed using Large-scale Atomic/Molecular Massively Parallel Simulator (LAMMPS) MD packages [55] and visualization was performed by Visual Molecular Dynamic (VMD) [56].

Table 3. L-J potential parameters for argon-argon and copper-copper

\begin{tabular}{lcc}
\hline & Argon-argon & Copper-copper \\
\hline$\varepsilon(\mathrm{J})$ & $1.6548 \times 10^{-21}$ & $65.6775 \times 10^{-21}$ \\
$\sigma(\mathrm{A})$ & 3.4050 & 2.3377 \\
\hline
\end{tabular}




\section{RESULTS AND DISCUSSION}

\subsection{System Validation}

4.1.1. Validation of base fluid with experimental data. In order to verify the methodology as well as the employed interatomic potential of liquid argon, validation with experimental data was carried out. Table 4 shows a comparison between the MD and experimental data $[57,58]$ on thermal conductivity and viscosity of argon liquid. The MD simulation provides data of error within $4.6 \%$ compared with experimental data. Thus, the EMD methodology and $\mathrm{L}-\mathrm{J}$ potential are confirmed as being capable to predict good results close to the experimental data, with a maximum relative error of $4.6 \%$.

4.1.2. Validation of equilibration process. There will be a nonequilibrium state for the initial setting of positions and velocities of atoms. The equilibration process is performed to insure the equilibrium state is achieved prior to the production of results. Therefore, the equilibrium state was validated by observing the convergence of temperature and total energy over the equilibration steps. Figure 2 shows that the temperatures of both systems fluctuated at the setting temperature of $86 \mathrm{~K}$. Convergence of temperature was achieved in both aggregated and nonaggregated states in nanofluids. From Figure 3, it will be observed that the total energy of systems converged and the insertion of copper nanoparticles increased the total energy of the argon liquid. The total energy of systems is increased by increasing the number of nanoparticles or volume fraction. A similar trend was also observed by Sankar et al. [59] in a MD simulation for nanofluids with a multinanoparticle system consisting of water and platinum. At a low volume fraction of $2.59 \%$, aggregated and nonaggregated states have similar total energies. When the volume fraction increased from $3.89 \%$ to $7.77 \%$, the total energy of the aggregated state was slightly higher than for the nonaggregated state.

4.1.3. Validation of HCACF. As discussed in Section 2, the area under HCACF refers to the thermal conductivity of system. HCACF must decay to zero within the integral time/correlation length $(M)$ to produce data convergence. Validation on the convergence of $\mathrm{HCACF}$ within a given integral time/correlation length was carried out. Figure 4 shows that the HCACF in both states decays well and achieves a plateau within the given correlation length of $20 \mathrm{ps}$. Therefore, the determination of correlation length of $20 \mathrm{ps}$ is sufficient to generate stable results for thermal conductivity. It is observed that the HCACF of argon liquid decayed to zero without any oscillation, and this is typical behavior for a liquid. Kaburaki [60] suggested that there are two regions of decay: the first region of rapid decay occurs at the beginning due to purely atomistic interaction, and the second region,

Table 4. Validation of thermal conductivity and viscosity of argon liquid with experimental data

\begin{tabular}{lccr}
\hline & MD data & Experimental data & Error \\
\hline Thermal conductivity $(\mathrm{W} / \mathrm{m} \cdot \mathrm{K})$ & 0.135 & $0.132[57]$ & $2.3 \%$ \\
Viscosity $(\mathrm{mPa} \cdot \mathrm{s})$ & 0.293 & $0.280[58]$ & $4.6 \%$ \\
\hline
\end{tabular}




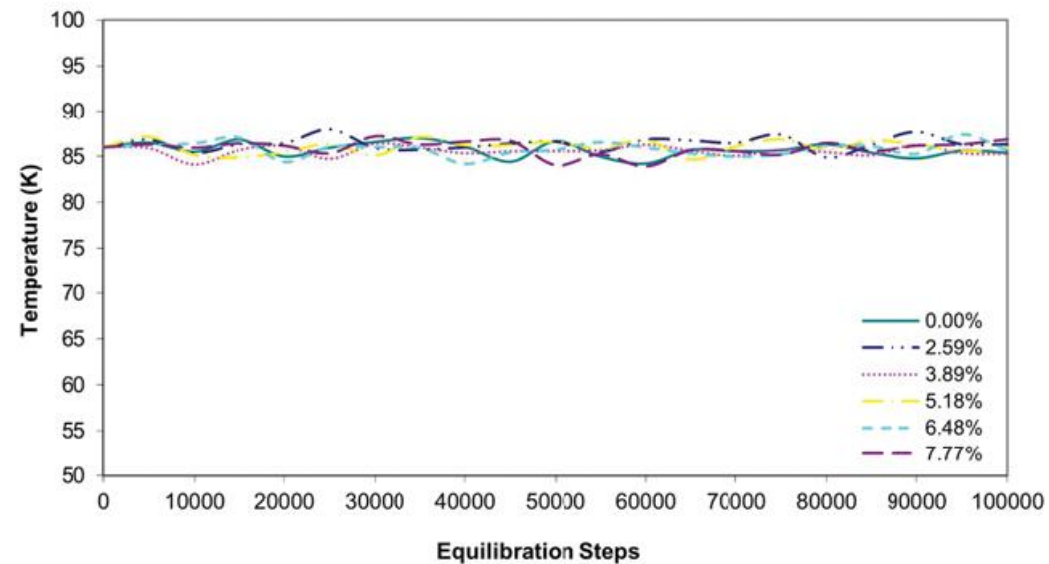

(a)

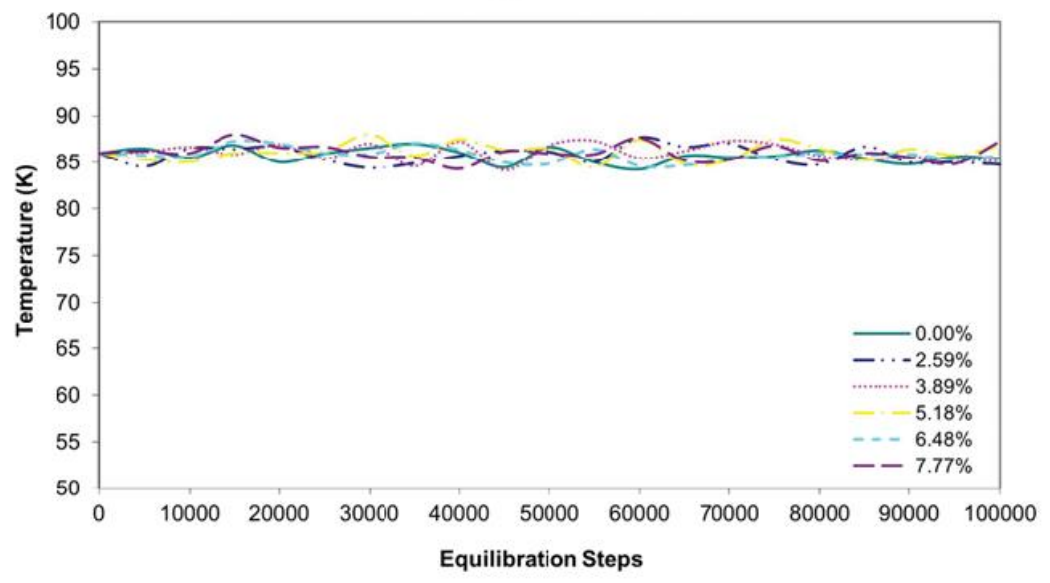

(b)

Figure 2. Temperature of base fluid and nanofluids during equilibration period. (a) Nonaggregated nanofluids, (b) aggregated nanofluids.

which is a larger region with slower decay, arises from phonon energy transfer. The presence of copper nanoparticles in base fluid changes the HCACF decay into an oscillation manner and exhibits a negative value. Such an oscillation manner is also observed in the crystalline and amorphous phases [61]. A similar oscillation manner in nanofluids was reported in MD simulation works [6, 43]. Keblinski et al. [6] indicated that a negative value of oscillations denotes that atoms are back scattered, phonons carrying heat energy are reflected by the solid-liquid interface, or phonons are trapped in a cage for short periods of time - the "cage effect." The insets in Figures $4 a$ and $b$ show that when nanoparticle volume fraction is increased, oscillation behavior becomes prominent resulting in an increase in thermal conductivity. Therefore, from the MD simulation it is observed that the thermal conductivity of 


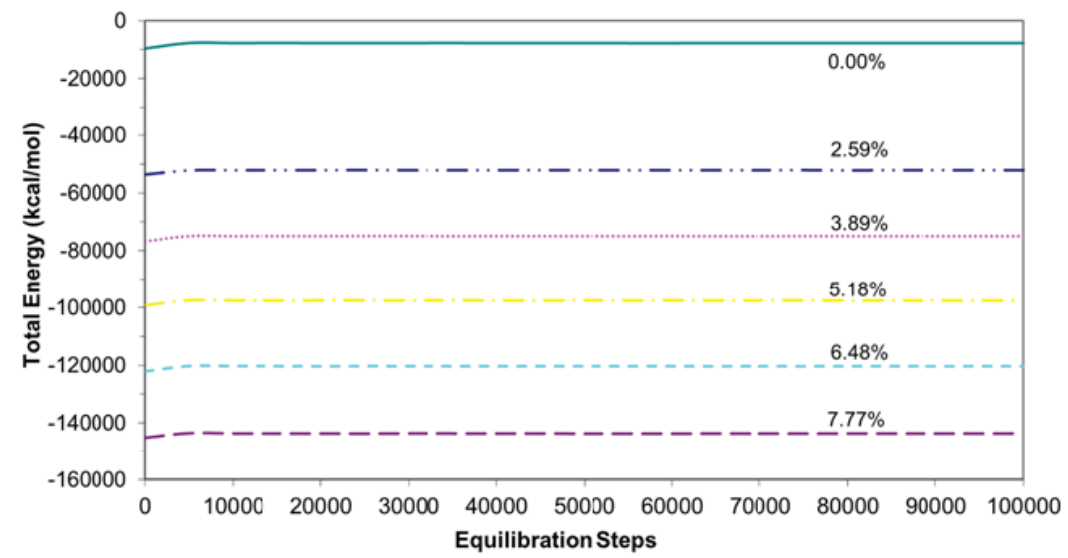

(a)

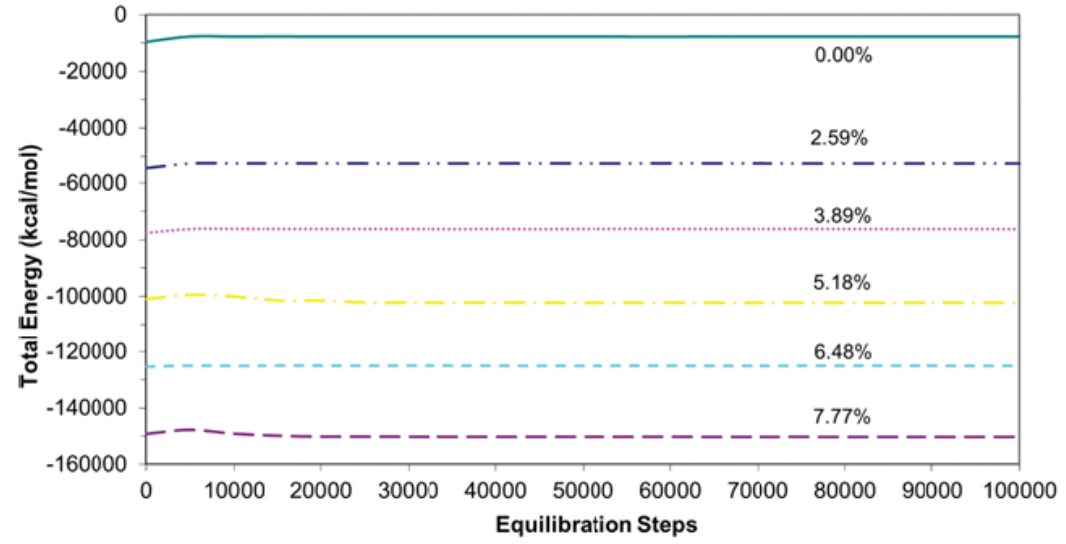

(b)

Figure 3. Total energy of base fluid and nanofluids during equilibration period. (a) Nonaggregated nanofluids, (b) aggregated nanofluids.

nanofluids is increased with increasing nanoparticle volume fraction. The presence of nanoparticles changes the structure of base fluid to an amorphous-like fluid structure and results in higher thermal conductivity.

\subsection{Calculation of Thermal Conductivity}

Thermal conductivities of nanofluids in aggregated and nonaggregated states are summarized in Figure 5. Thermal conductivity according to the prediction of Maxwell is also shown for comparison. The thermal conductivity of both states increases with increasing nanoparticle volume fraction and is higher than the prediction of Maxwell. For aggregated nanofluids, thermal conductivity increased from 0.185 to 0.196 , to 0.218 , to 0.235 , and then to $0.255 \mathrm{~W} / \mathrm{m} \cdot \mathrm{K}$ for nanoparticle volume fraction increments from $2.59 \%$ to $3.89 \%$, to $5.18 \%, 6.48 \%$, and then to $7.77 \%$. Such 
Link to full text journal article :

http://www.tandfonline.com/doi/pdf/10.1080/10407782.2014.986366 